() OPEN ACCESS

\title{
Comparison of clinical outcomes of conbercept versus ranibizumab treatment for retinopathy of prematurity: a multicentral prospective randomised controlled trial
}

\author{
Zhenquan $\mathrm{Wu}^{1}{ }^{1}$ Jinfeng Zhao $\bullet$, ${ }^{1}$ Waiching Lam, ${ }^{2}$ Mingmin Yang, ${ }^{1}$ Lu Chen ${ }^{\circ},{ }^{1}$ \\ Xuelin Huang, ${ }^{3}$ Meirong Wei, ${ }^{4}$ Hui Yang ${ }_{1}^{5}$ Fan Lv ${ }_{1}^{6}$ Fuyan Zhang, ${ }_{1}^{7}$ Jian Zeng, ${ }_{1}{ }^{1}$ \\ Guo-Ming Zhang ${ }^{1}$
}

\begin{abstract}
${ }^{1}$ Ophthalmology, Shenzhen Eye Hospital, Shenzhen Key Laboratory of Ophthalmology, Shenzhen University School of Medicine, Shenzhen, Guangdong, China, Shenzhen, Guangdong, China

${ }^{2}$ Ophthalmology, The University of Hong Kong, Grantham Hospital, Hong Kong, China ${ }^{3}$ Ophthalmology, Guangdong Provincial Maternity and Children's Hospital, Guangzhou, Guangdong, China

${ }^{4}$ Ophthalmology, Liuzhou Maternity and Children's Hospital, Liuzhou, China ${ }^{5}$ Ophthalmology, Xiamen Children's Hospital, Xiamen, China

${ }^{6}$ Ophthalmology, Quanzhou Children's Hospital, Quanzhou, China

${ }^{7}$ Ophthalmology, Guizhou Medical University, Guiyang, Guizhou, China
\end{abstract}

\section{Correspondence to} Dr Guo-Ming Zhang, ophthalmology, Shenzhen Eye Hospital, Shenzhen 518040, Guangdong, China;

\section{0@163.com}

Received 19 September 2020 Revised 24 January 2021 Accepted 11 February 2021
Check for updates

(C) Author(s) (or their employer(s)) 2021. Re-use permitted under CC BY-NC. No commercial re-use. See rights and permissions. Published by BMJ.

\section{To cite: Wu Z, Zhao J,} Lam W, et al. Br J Ophthalmol Epub ahead of print: [please include Day Month Year]. doi:10.1136/

bjophthalmol-2020-318026

\section{ABSTRACT}

Purpose To compare the recurrence rate and surgical complications of retinopathy of prematurity (ROP) between patients treated with intravitreal injection of conbercept (IVC) and intravitreal injection of ranibizumab (IVR) within 6 months.

Methods A multicentral prospective, randomised controlled trial was applied from May 2017 to February 2019 for the infants diagnosed as aggressive posteriorROP, zone I or posterior zone II treatment-requiring ROP by binocular indirect ophthalmoscope and RetCam3. These infants were assigned to randomly receive either intravitreal injection of $0.25 \mathrm{mg}$ conbercept or $0.25 \mathrm{mg}$ ranibizumab. The recurrence rate, fundus fluorescence angiography (FFA) and surgical complications were examined during the follow-up period of 6 months. Recurrent eyes were retreated by laser or another intravitreal injection within the 72 hours.

Results A total of 30 infant patients (60 eyes) underwent IVC and 30 patients (60 eyes) underwent IVR. A total of 10 eyes $(16.67 \%)$ in the IVC group and 14 eyes $(23.34 \%)$ in the IVR group developed recurrence. There was no significant statistical difference in the recurrence rate between the two groups $\left(\chi^{2}=0.83\right.$, $p=0.36)$. The postmenstrual age (PMA) at first injection was (34.60 \pm 3.47$)$ weeks in IVC and (35.14 \pm 1.76$)$ in IVR group. In recurrent cases, the mean PMA at second treatment were $(43.31 \pm 3.85)$ and $(43.43 \pm 3.89)$ weeks in the IVC and IVR group, respectively. The period between two treatments was $(8.71 \pm 6.62)$ for the IVC and $(8.29 \pm 2.56)$ weeks for the IVR group. All these results showed no significant statistical difference between these two groups. The fluorescein leakage were observed in the eyes of recurrent infants by FFA. There were no other complications in the two groups except for complicated cataract in three eyes.

Conclusion Both IVC and IVR are effective therapies for the treatment of ROP. Conbercept is a new option for treating ROP.

\section{INTRODUCTION}

Retinopathy of prematurity (ROP) is the major cause for childhood blindness worldwide. ${ }^{12}$ The vascular endothelial growth factor (VEGF) is regarded as a main factor leading to $\mathrm{ROP}^{3}$ The dysregulation of VEGF associated with hypoxia is thought to play an significant role in its abnormal vasculogenesis and neovascularisation. ${ }^{4}$ The traditional standard treatment for ROP is laser photocoagulation (LP).$^{5-7}$ But the side effect of LP should not be ignored including visual field loss and subsequent high myopia. ${ }^{4}$ In recent years, anti-VEGF treatment has become a new effective therapy for ROP. $^{489}$ Compared with LP, anti-VEGF agents have the advantages including short operating time, no need of sedation or general anaesthesia and no ablation of peripheral retina. ${ }^{10-12}$

There have been some studies comparing the efficacy of different anti-VEGF agents in the treatment of ROP, such as conbercept and ranibizumab, ranibizumab and aflibercept, bevacizumab and ranibizumab. ${ }^{41-15}$ Some of these anti-VEGF agents have been used by experts in treating ROP for many years, while conbercept (KH902; Chengdu Kanghong Biotech, Sichuan, China), as a new anti-VEGF agent, has been used in China just in recent years. Conbercept was first approved by China Food and Drug Administration for intravitreal injection in treating age-related macular degeneration (AMD) in 2013. It is a recombinant fusion protein. And it has the characteristics of multiple targets, stronger affinity and the ability to inhibit neovascularisation. The safety and efficacy of injection of conbercept for AMD and ROP have been confirmed by retrospective studies. ${ }^{4} 16$

As we described above, the safety and efficacy of various anti-VEGF agents for ROP have been confirmed by many studies. ${ }^{43-15}$ The researches of these studies have shown that the anti-VEGF agents are effective for ROP. But most of these studies were retrospective. Prospective studies in this field to further investigate the efficacy of defferent anti-VEGF agents are particularly important. At present, few prospective studies have been reported in comparison of conbercept and ranibizumab in treating ROP.

This study aims to compare the recurrence rates and related outcomes between intravitreal injection of conbercept (IVC) and intravitreal injection of ranibizumab (IVR) in ROP treatment through a prospective randomised controlled trial (RCT), which might provide insights of ROP treatment for clinical ophthalmologists.

\section{METHODS \\ Examination and enrolment}

A total of 60 infants of patients with ROP were consecutively enrolled in this study from various 
centres and hospitals nationwide during the period of May 2017 to February 2019. They included Shenzhen Eye Hospital, Guangdong Provincial Maternity and Children's Hospital, Liuzhou Maternity and Children's Hospital, Xiamen Children's Hospital and Quanzhou Children's Hospital. We employed a multicentral prospective RCT in this study. All the infants were examined via binocular indirect ophthalmoscope and fundus imaging by RetCam 3 (Clarity Medical System, Pleasanton, California, USA) at the first and each follow-up visit. Each infant was examined by two experienced retinal experts independently, and the eligibility was confirmed by both experienced retinal experts. The definition of stage and zone was based on the revised guidelines of the International Committee for the Classification of Retinopathy of Prematurity. ${ }^{17}$ The definition of treatment requiring ROP were based on the Early Treatment ROP. ${ }^{618}$ Inclusion criteria contain: (1) aggressive posterior ROP (AP-ROP); (2) Zone I or posterior zone II treatment-requiring ROP (any stage of ROP with plus disease or stage three without plus disease in zone I. Stages 2 and 3 with plus disease in zone II.); (3) agreement of parents to participate in the trial. Exclusion criteria include: (1) infants with other eye diseases; (2) infants with any previous treatment including intravitreal injection, LP, cryotherapy or other ocular surgery.

\section{Study design}

All eligible infants were randomly divided into two groups at a 1:1 proportion using a computer-generated randomisation schedule. Both eyes of each infant were then treated by either the IVC or IVR herapy. The primary observational indicators was the recurrence rate. Other observational indicators were retinal features of recurrence based on the fundus fluorescence angiography (FFA) and the complications after the treatment. The participants' parents were informed about the severity of the disease, treatment options and complications. And this study obtained the informed consent of the parents before the study was performed.

\section{Treatment}

All the included infants in the IVC group received an IVC (Conbercept; Chengdu Kanghong Biotech, China) with a dose of $0.25 \mathrm{mg} / 0.025 \mathrm{~mL}$ half of the adult dosage. For the IVR group, all the infants received an intravitreal injection of ranibizumab (Lucentis; Genentech and Novartis International AG) with the same dose of $0.25 \mathrm{mg} / 0.025 \mathrm{~mL}$. Intravitreal injection was performed under topical anaesthesia in the standard ophthalmic operating room. This anti-VEGF agent was injected into the vitreous, pointing the needle toward the optic nerve in direction of the visual axis $1.0 \mathrm{~mm}$ posterior to the corneoscleral junction. An ophthalmic antibiotic eye-drop was prescribed for the treated eye four times a day for 1 week. Another intravitreal injection (IVI) or LP would be arranged as a secondary treatment when recurrence occurred. The application of anti-VEGF agents is suitable for the recurrent infants whose lesions are close to the posterior pole of retina, and LP is more appropriate for infants with lesions near the periphery. An indirect infrared diode laser (Iridis; Quantel-Medical, Cournon d'Auvergne Cedex, France) $(810 \mathrm{~nm})$ was used to apply photocoagulation through a 20 -diopter condensing len under the state of sedation. The procedure of sedation was peformed by an experienced nurse in the neonatal intensive care unit using phenobarbital and diazepam intravenous injection. The sedative strategy was determined by neonatologists based on the weight of infants. Initial laser was set up at a power of $150 \mathrm{~mW}$ for $0.2 \mathrm{~s}$. Confluent laser treatment was applied to the avascular retina between the fibrovascular ridge and the ora serrata. The laser treatment for peripheral retina was performed under scleral indentation. The antibiotic eye-drop was administrated for 1 week after laser treatment.

\section{Follow-up}

All infants were monitored by binocular indirect ophthalmoscope and RetCam 3 after the treatment following the timeline: $1,2,4,6,8,10,12,18$ and 24 weeks after the treatment. The follow-up occured at least 6 months. The main outcome measurements included the regression of plus disease, resolution of neovascularisation, disappearance of ridge, ROP recurrences and any complications. The recurrence was defined as any of the following: recurrent plus disease, recurrent neovascularisation or reformation of ridge despite treatment. Once the recurrence occurred, the secondary treatment was arranged for retreatment within 72 hours. $^{19}$

\section{Statistical analyses}

Statistical analysis was performed by the SPSS software (V.23). Normally distributed variables were compared and constrasted by the t-test while the $\chi^{2}$ test was used to examine categorical variables. The $\mathrm{p}<0.05$ was considered statistically significant in this study.

\section{RESULTS}

A total of 60 eyes of 30 infants were treated with IVC monotherapy, and 60 eyes of another 30 infants were treated with IVR monotherapy. These two groups of infants have completed the 6-month follow-up. Our results show that there was no statistically significant difference in gestational age (GA), birth weight (BW), sex ratio, the proportion of single or twin births, delivery methods and corrected GA at first treatment between two groups (table 1). And in the IVC group, the medians of BW, GA and postmenstrual age (PMA) were $1047.5 \mathrm{~g}$ (600-2500 g), 28.43 weeks (24-32.86 weeks) and 35 weeks (29.43-39.43 weeks), respectively. In the IVR group, the medians of BW, GA and PMA were $975 \mathrm{~g}(590-1930 \mathrm{~g}), 27$ weeks $(24-33.14 \mathrm{w})$ and 35.79 weeks (32-42 weeks), respectively.

\section{Main outcomes}

After a single-dose injection, a total of 10 eyes of 5 infants (16.7\%) developed ROP recurrence in the IVC group, and 14 eyes of 7 infants $(23.34 \%)$ developed ROP recurrence in the IVR counterpart. There was no significant difference between the two groups $(\chi 2=0.83, p=0.36)$. All of the recurrent infants in the IVC group and five recurrent infants in the IVR group were

Table 1 The characteristics of infants in the IVC and IVR groups

\begin{tabular}{llllll}
\hline & IVC group & IVR group & T & $\chi^{2}$ & P value \\
\hline No of eyes/children & $60 / 30$ & $60 / 30$ & $/$ & & / \\
\hline Male/female & $16 / 14$ & $17 / 13$ & $/$ & 0.07 & $0.795^{*}$ \\
Single/ multiple & $22 / 8$ & $26 / 4$ & $/$ & 1.67 & $0.196^{*}$ \\
NB/CS & $16 / 14$ & $20 / 10$ & $/$ & 1.11 & $0.292^{*}$ \\
BW (g) & $1160 \pm 480$ & $1089 \pm 400$ & 0.59 & 0.558 \\
GA (week) & $28.27 \pm 2.77$ & $27.50 \pm 2.70$ & 1.08 & 0.284 \\
$\begin{array}{l}\text { PMA at first treatment } \\
\text { (week) }\end{array}$ & $35.26 \pm 1.91$ & $36.08 \pm 2.25$ & -1.52 & & 0.133 \\
\hline
\end{tabular}

${ }^{*} \chi^{2}$ test. The remaining indices are the $t$-test.

BW, birth weight; CS, caesarean section; GA, gestational age; IVC, intravitreal injection of conbercept; IVR, intravitreal injection of ranibizumab; NB, natural birth; PMA, postmenstrual age. 

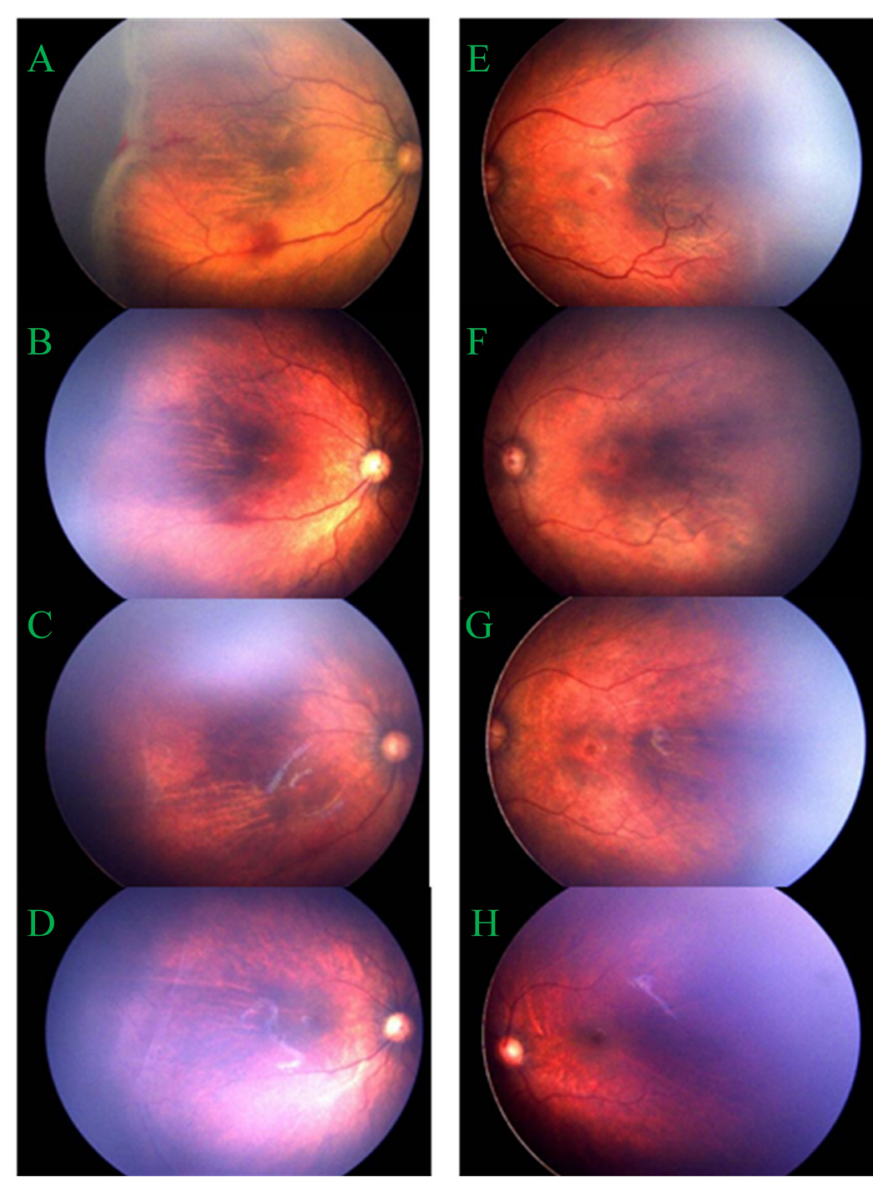

Figure 1 Fundus images of no recurrence cases. (A-D) fundus images in the IVC group. $(E-H)$ fundus images in the IVR group. $(A, E)$ images before IVI shows plus disease and ridge. $(B, F)$ regression of plus disease and ridge was observed at 1 week after treatment. $(C, G)$ one month after treatment. $(\mathrm{D}, \mathrm{H})$ six months after treatment. No recurrence occurred during the 6 months follow-up. IVC, intravitreal injection of conbercept; IVI, intravitreal injection; IVR, intravitreal injection of ranibizumab.

treated with the LP, and the remaining two recurrent infants in the IVR group were treated with the IVR. For the infants with and without recurrence, we selected a series of representative images to show the results of each examination. figure $1 \mathrm{~A}-\mathrm{H}$ shows the fundus images in the IVC and IVR group, respectively, which represented cases without recurrence. figure $2 \mathrm{~A}-\mathrm{J}$ shows the fundus images in the IVC and IVR group, respectively, which represented cases with recurrence.

For the recurrent infants, the PMA at first treatment reached $34.60 \pm 3.47$ weeks in the IVC group and $35.14 \pm 1.7$ weeks in the IVR counterpart. There was no statistically significant difference in both groups $(t=-0.36, p=0.728)$. The PMA at second treatment in these two groups were $43.31 \pm 3.85$ and $43.43 \pm 3.89$ weeks. Likewise, there was no statistically significant difference in both groups $(t=-0.05, p=0.961)$. The interval of these two treatments in the two groups was 8.71 \pm 6.62 and $8.29 \pm 2.56$ weeks. Again, there was no statistically significant difference in both groups $(t=-0.16, p=0.878)$. Details of the infants were given in the tables 1 and 2 .

We observed the total regression of plus disease and ridge in all recurrent infants after the secondary treatment, and there was no new recurrence occurred during the follow-up period (figure 2A-J).
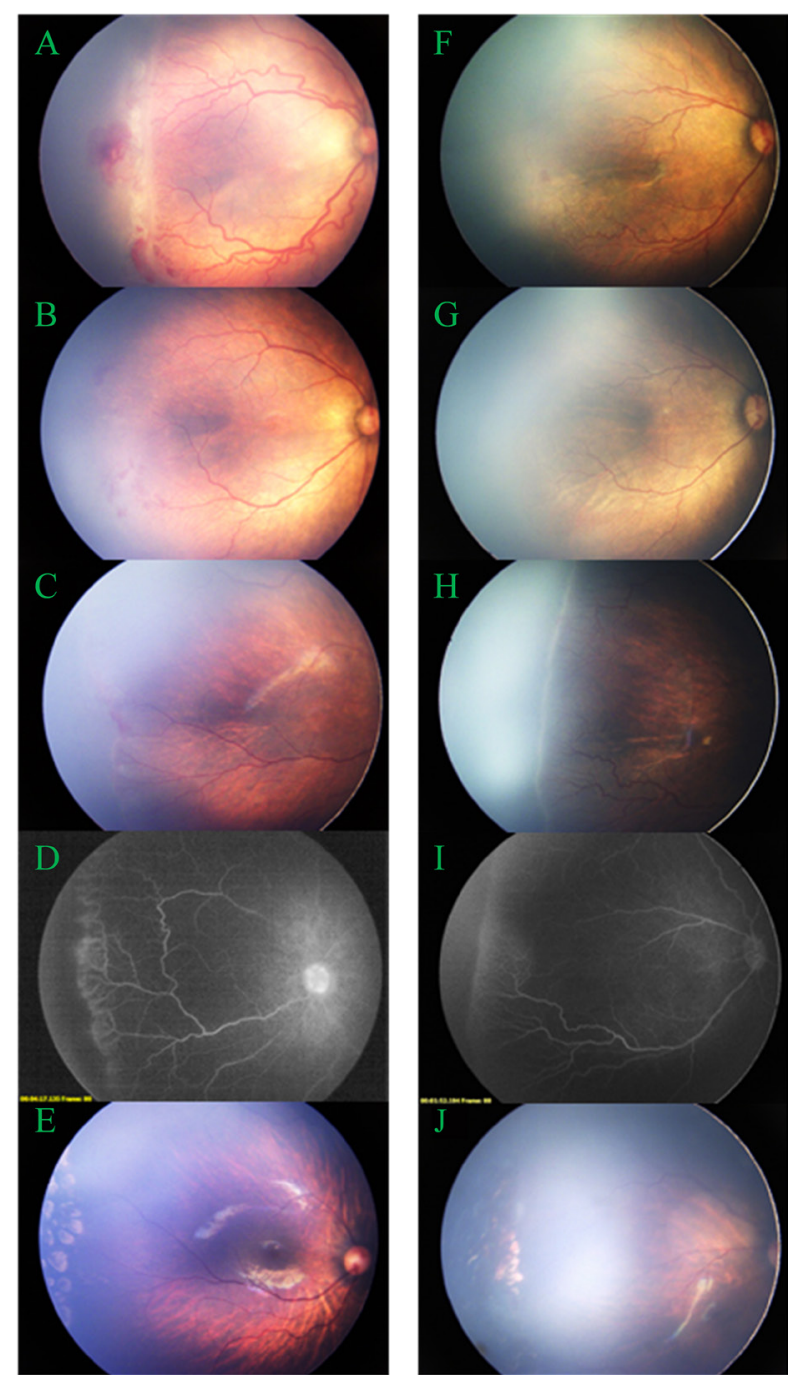

Figure 2 Fundus images of recurrence cases. (A-E) fundus images in the IVC group. (F-J) fundus images in the IVR group. (A,F) Images before IVI shows plus disease and ridge. $(B, G)$ Regression of plus disease and ridge was observed at 1 week after treatment. $(\mathrm{C}, \mathrm{H})$ aggravated plus disease and ridge was observed during follow-up. $(D, I)$ FFA shows the retinal neovascularisation and fluorescence. $\left(E_{,} J\right)$ six months after IVI. No new recurrence was observed after secondary treatment. FFA, fundus fluorescence angiography; IVC, intravitreal injection of conbercept; IVR, intravitreal injection of ranibizumab.

\section{Features of recurrence based on FFA}

The FFA examination was performed in 5 recurrent infants (10 eyes) before the second treatment. Their vessels in the posterior pole are seen to be tortuous and dilated. There is a wide avascular area in the peripheral retina. The ROP ridge aggravated. The plexiform arteriovenous anastomosis occurred. The branches of peripheral vessels increased, presenting a 'brush-like' change. The vascular blunt, vascular loops and the neovascularisation were observed, accompanied by obvious fluorescence leakage, presenting a 'popcorn-like' change (figure 2D,I).

\section{Complications}

A total of three eyes (ie, two in the IVC and one in the IVR group) generated complicated cataracts after treatment. No anterior segment ischemia, pupillary membrane, retinal detachment, endophthalmitis, glaucoma, intraocular haemorrhage or 


\begin{tabular}{|c|c|c|c|c|c|}
\hline & IVC group & IVR group & $T$ & $\chi^{2}$ & $P$ value \\
\hline Recurrent eyes/patient & $10 / 5$ & $14 / 7$ & I & 0.83 & 0.36 \\
\hline Recurrence rate & $16.67 \%$ & $23.34 \%$ & I & I & I \\
\hline $\begin{array}{l}\text { PMA at initial treatment in } \\
\text { recurrent infant }(w)\end{array}$ & $34.60 \pm 3.47$ & $35.14 \pm 1.76$ & -0.36 & I & 0.728 \\
\hline $\begin{array}{l}\text { PMA at second treatment in } \\
\text { recurrent infant }(w)\end{array}$ & $43.31 \pm 3.85$ & $43.43 \pm 3.89$ & -0.05 & 1 & 0.961 \\
\hline $\begin{array}{l}\text { Interval between two } \\
\text { treatment(w) }\end{array}$ & $8.71 \pm 6.62$ & $8.29 \pm 2.56$ & 0.16 & 1 & 0.878 \\
\hline
\end{tabular}

IVC, intravitreal injection of conbercept; IVR, intravitreal injection of ranibizumab; PMA, postmenstrual age.

systemic complications related to injection occurred during the follow-up period.

\section{DISCUSSION}

The LP is a conventional treatment for ROP. ${ }^{617}$ The mechanism of LP is known to suppress VEGF by ablating the avascular retina. However, LP shows several limitations, such as ametropia and defection of the visual field. ${ }^{19}$ Compared with LP, anti-VEGF therapy is more convenient to operate and shows no irreversible damage to the retina. It has been regarded as a primary monotherapy for zone I ROP and AP-ROP. ${ }^{10-12}$ Recently, anti-VEGF therapy has been increasingly applied in ROP treatment. The conbercept and ranibizumab have been reported and proved to be effective. ${ }^{4}$ Previous research has compared the curative effect of IVC and IVR in the treatment of ROP. ${ }^{4}$ But most of studies were retrospective, and the sample size is relatively small. This study is the multicentral prospective RCT of conbercept versus ranibizumab for ROP, which might provide insights from a new perspective for clinical ophthalmologists.

The results on the IVC recurrence rate showed great similarities in previous studies. For example, Bai et al ${ }^{16}$ enrolled 24 patients (48 eyes) who received $0.25 \mathrm{mg}$ IVC treatment and a follow-up for more than 6 months. The authors showed that a total of 8 eyes $(16.7 \%)$ required secondary treatment. In Jin's retrospective study, the recurrence rate was $15 \%(3 / 20)$ in the IVC group but $53.6 \%(15 / 28)$ in the IVR counterpart. ${ }^{4}$ In our study, the recurrence rate of IVC is $16.7 \%(10 / 60)$. Recurrent ROP was treated by LP and obtained regression. This conclusion generally agrees with those in previous studies.

Conbercept, as a new anti-VEGF agent, is a fusion protein which combines the second Ig-like domain of VEGFR-1 and the third and fourth Ig-like domains of VEGFR-2 to the Fc portion of human IgG1. Thus, it has the characteristics of multiple targets, stronger affinity (such as VEGF-A, VEGF-B, VEGF-C and PIGF), and the ability to inhibit neovascularisation. ${ }^{20-24}$ Domain 4 of the conbercept can reduce positive charge, reduce adhesion of extracellular matrix, optimise pharmacokinetics and make it have stronger affinity and longer half-life. ${ }^{21}$ The use of conbercept for ROP has been reported. ${ }^{162526}$ Cheng et al $^{26}$ used a lower dose $(0.15 \mathrm{mg} / 0.015 \mathrm{~mL})$ of conbercept for ROP, and the recurrence rate was reported to be $15.8 \%$. This suggested that a low dose of conbercept was also effective in ROP, which might reduce the potential systemic risks.

However, the IVR recurrence rate varied greatly from $0 \%$ to more than $80 \%$ in previous retrospective studies. Ranibizumab is a specific, recombinant, humanised monoclonal antibody with high binding affinity for VEGF-A, rendering the VEGF inactive. As for the different results of different studies, we consider that the different inclusion criteria with various researches, the subjectivity of different experts in diagnosis, recurrence definition, timing of operation and follow-up schedules may cause the diversity simultaneously. In this study, there is no significant statistical difference in the recurrence rate between the IVC and IVR groups. More importantly, this was a prospective RCT of conbercept vs ranibizumab with a relatively high level of evidence. Our inclusion criteria and intervention are more unified, and the evaluation of these two therapies is more objective and accurate.

For the timing of recurrence, previous research reported the time of 4-8 weeks after IVR or approximately 41-42 weeks' PMA. ${ }^{27-29}$ In Lyu's study, the recurrence of ROP occurred in 32 $(64 \%)$ of 50 eyes at $7.9 \pm 2.7$ weeks after IVR, and the major recurrence risk period was from 2.5 to 12.0 weeks after IVR, with its risk peak at 8 weeks. In our study, however, the interval of ROP in the IVC and IVR group reached $8.71 \pm 6.62$ weeks and $8.29 \pm 2.56$ weeks, respectively. A total of $91.67 \%(22 / 24)$ recurrence occurred within 12 weeks after IVC or IVR. Based on our study and previous reports, a close follow-up in the first 12 weeks after treatment is indispensable for timely identification of ROP recurrence. However, the one infant in the IVR group in this studiy had a long interval (20.28 weeks) between two treatments with an earlier GA and initial treatment time. As a result, a longer follow-up should be arranged for such infants with late recurrence in case of misdiagnosis. According to previous studies and the results of this study, ${ }^{30}$ a follow-up longer than 24 weeks after IVI would be better for management of ROP recurrence. The optimal follow-up time, if available, still needs to be further verified by long-term follow-ups with a larger sample size. In this study, half of the infants with recurrence were diagnosed with AP-ROP. This suggests that infants with severe disease are more likely to be recurrent. Although some cases of recurrence occurred, most of them were well controlled after the second treatment. ${ }^{16}$

The FFA, as an adjunct to indirect ophthalmoscope, is an important technique to identify the vascular changes of ROP. ${ }^{3132}$ In this study, FFA was performed on certain infants with recurrence before secondary treatment, as this technology distinctly showed the vascular abnormalities, such as vascular blunt, loops and dilatation, and leakage. The leakage is a significant feature suggesting neovascularisation, which provides a convincing evidence for recurrence. Compared with direct observation, FFA is more sensitive for demarcation between vascular and avascular areas. After IVI treatment, we observed that retinal blood vessels could continue to develop to zone III, but not all infants' vessels could reach the ora serrata. The effects of the avascular areas remain unknown. How to deal with the avascular area after IVI treatment is still debatable, and thus long-tern observation and supplementing FFA are required. Owing to the limitation of equipment in certain centres, FFA was employed only in 5 infants (10 eyes) in this study.

Complicated cataract occurred in three eyes, characterised by a single localised punctate opacity on peripheral lens. This complication might be iatrogenic damage caused by the fact that the needle touched the lens during the surgery, indicating that sufficient topic anaesthesia should be undergone before the treatment. Surgical light and eyeball fixation should be ensured during the operation. The $1-1.5 \mathrm{~mm}$ posterior to the corneal limbus should be the entry point. The needle should be oriented towards the centre of the eyeball to reduce the risk of iatrogenic injury. For these infants who have developed cataracts, the opacity may not affect visual development because of the peripheral location, but more future observation is necessary. 


\section{Limitations}

First, the sample size (ie, 30 infants for IVC and 30 for VR) is relatively small and the follow-up (ie, 6 months) is not so long. More patients should be included to increase the conviction in further studies, and the follow-up duration should be prolonged too. Second, we did not evaluate the systematic effects of two medicines on infants. The comparison of preoperative and postoperative blood VEGF levels in infants is of great significance for the safety evaluation, which needs to be supplemented in further research.

\section{Conclusion}

In conclusion, there was no significant statistical difference in the recurrence rate between IVC and IVR for treating ROP within 6 months. Both of the IVC and IVR are effective treatments for ROP. Conbercept is a new option for ROP treatment.

\section{Acknowledgements The authors would like to thank all the patients who participated in this study.}

Contributors ZW and JZ contributed equally to this work. JZ and ZW contributed to the conception of the study. JZ, ZW and G-MZ performed the data analyses and wrote the manuscript. WL, JZ and FZ helped perform the analysis with constructive discussions. MY and LC contributed significantly to analysis and manuscript preparation. XH, MW, HY and FL in the Shenzhen Screening for Retinopathy of Prematurity Cooperative Group provided multicentre cases.

Funding This work was supported by the Basic Discipline Layout Foundation of the Shenzhen Science and Innovation Commission. P.R. China (Grant No. JCYJ20170817112542555); Shenzhen-hong Kong Cofunded Projects (Category A): SGDX20190920110403741.

Competing interests None declared.

Patient consent for publication Not required.

Ethics approval This protocol was approved by the Institutional Review Board of Shenzhen Eye Hospital, which was conducted in accordance with the Declaration of Helsinki.

Provenance and peer review Not commissioned; externally peer reviewed.

Data availability statement Data are available in a public, open access repository. Data are available on reasonable request.

Open access This is an open access article distributed in accordance with the Creative Commons Attribution Non Commercial (CC BY-NC 4.0) license, which permits others to distribute, remix, adapt, build upon this work non-commercially, and license their derivative works on different terms, provided the original work is properly cited, appropriate credit is given, any changes made indicated, and the use is non-commercial. See: http://creativecommons.org/licenses/by-nc/4.0/.

\section{ORCID iDs}

Jinfeng Zhao http://orcid.org/0000-0003-1422-5203

Lu Chen http://orcid.org/0000-0003-1141-8288

\section{REFERENCES}

1 Fierson WM, et al, American Academy of Pediatrics Section on Ophthalmology, American Academy of Ophthalmology. Screening examination of premature infants for retinopathy of prematurity. Pediatrics 2018;142:e20183061.

2 Blencowe H, Lawn JE, Vazquez T, et al. Preterm-associated visual impairment and estimates of retinopathy of prematurity at regional and global levels for 2010. Pediat Res 2013;74 Suppl 1:35-49.

3 Hartnett ME, Penn JS. Mechanisms and management of retinopathy of prematurity. N Engl J Med 2013;368:239-43.

4 Jin E, Yin H, Li X, et al. Short-Term outcomes after intravitreal injections of CONBERCEPT versus ranibizumab for the treatment of retinopathy of prematurity. Retina 2018:38:1595-604.

5 Palmer EA. Results of U.S. randomized clinical trial of cryotherapy for ROP (CRYOROP). Doc Ophthalmol 1990:74:245-51.

6 Good WV, Early Treatment for Retinopathy of Prematurity Cooperative Group. Final results of the early treatment for retinopathy of prematurity (ETROP) randomized trial. Trans Am Ophthalmol Soc 2004;102:233-48.
7 Geloneck MM, Chuang AZ, Clark WL, et al. Refractive outcomes following bevacizumab monotherapy compared with conventional laser treatment: a randomized clinical trial. JAMA Ophthalmol 2014;132:1327-33.

8 Mintz-Hittner HA, Kennedy KA, Chuang AZ. Efficacy of intravitreal bevacizumab for stage 3+ retinopathy of prematurity. $N$ Engl I Med Overseas Ed 2011;364:197:603-15.

9 Arámbulo O, Dib G, Iturralde J, et al. Intravitreal ranibizumab as a primary or a combined treatment for severe retinopathy of prematurity. Clin Ophthalmol 2015;9:2027-32.

10 Feng J, Qian J, Jiang Y, et al. Efficacy of primary intravitreal ranibizumab for retinopathy of prematurity in China. Ophthalmology 2017;124:408-9.

11 Menke MN, Framme C, Nelle M, et al. Intravitreal ranibizumab monotherapy to treat retinopathy of prematurity zone II, stage 3 with plus disease. BMC Ophthalmol 2015; $15: 20$

12 VanderVeen DK, Melia M, Yang MB, et al. Anti-Vascular endothelial growth factor therapy for primary treatment of type 1 retinopathy of prematurity: a report by the American Academy of ophthalmology. Ophthalmology 2017;124:619-33.

13 Sukgen EA, Koçluk Y. Comparison of clinical outcomes of intravitreal ranibizumab and aflibercept treatment for retinopathy of prematurity. Graefes Arch Clin Exp Ophthalmol 2019;257:49-55.

14 Kimyon S, Mete A. Comparison of bevacizumab and ranibizumab in the treatment of type 1 retinopathy of prematurity affecting zone 1. Ophthalmologica 2018;240:99-105.

15 Chen Y-C, Chen S-N, Yang BC-L, et al. Refractive and biometric outcomes in patients with retinopathy of prematurity treated with intravitreal injection of ranibizumab as compared with bevacizumab: a clinical study of correction at three years of age. J Ophthalmol 2018;2018:1-6

16 Bai Y, Nie H, Wei S, et al. Efficacy of intravitreal conbercept injection in the treatment of retinopathy of prematurity. Br J Ophthalmol 2019;103:494-8.

17 International Committee for the Classification of Retinopathy of Prematurity. The International classification of retinopathy of prematurity revisited. Arch Ophthalmol 2005;123:991-9.

18 Early Treatment For Retinopathy Of Prematurity Cooperative Group. Revised indications for the treatment of retinopathy of prematurity: results of the early treatment for retinopathy of prematurity randomized trial. Arch Ophthalmol 2003;121:1684-94.

19 Zhang G, Yang M, Zeng J, et al. Comparison of intravitreal injection of ranibizumab versus laser therapy for zone II treatment-requiring retinopathy of prematurity. Retina 2017:37:710-7.

$20 \mathrm{Wu}$ Z, Zhou P, Li X, et al. Structural characterization of a recombinant fusion protein by instrumental analysis and molecular modeling. PLoS One 2013;8:e57642

21 Lu X, Sun X. Profile of conbercept in the treatment of neovascular age-related macular degeneration. Drug Des Devel Ther 2015;9:2311-20.

22 Zhang $\mathrm{M}$, Zhang J, Yan $\mathrm{M}$, et al. Recombinant anti-vascular endothelial growth factor fusion protein efficiently suppresses choridal neovasularization in monkeys. Mol Vis 2008;14:37-49.

23 Zhang M, Yu D, Yang C, et al. The pharmacology study of a new recombinant human VEGF receptor-Fc fusion protein on experimental choroidal neovascularization. Pharm Res 2009:26:204-10.

24 Gaudreault J, Fei D, Beyer JC, et al. Pharmacokinetics and retinal distribution of ranibizumab, a humanized antibody fragment directed against VEGF-A, following intravitreal administration in rabbits. Retina 2007;27:1260-6.

25 Li X, Xu G, Wang Y, et al. Safety and efficacy of conbercept in neovascular age-related macular degeneration: results from a 12-month randomized phase 2 study: Aurora study. Ophthalmology 2014;121:1740-7.

26 Cheng Y, Meng Q, Linghu D, et al. A lower dose of intravitreal conbercept effectively treats retinopathy of prematurity. Sci Rep 2018;8:10732.

27 Chan JJT, Lam CPS, Kwok MKM, et al. Risk of recurrence of retinopathy of prematurity after initial intravitreal ranibizumab therapy. Sci Rep 2016;6:27082.

28 Wong RK, Hubschman S, Tsui I. Reactivation of retinopathy of prematurity after ranibizumab treatment. Retina 2015;35:675-80

29 Yi Z, Su Y, Zhou Y, et al. Effects of intravitreal ranibizumab in the treatment of retinopathy of prematurity in Chinese infants. Curr Eye Res 2016;41:1092-7.

30 Lyu J, Zhang Q, Chen C-L, et al. Recurrence of retinopathy of prematurity after intravitreal ranibizumab monotherapy: timing and risk factors. Invest Ophthalmol Vis Sci 2017;58:1719-25

31 Lepore D, Quinn GE, Molle F, et al. Intravitreal bevacizumab versus laser treatment in type 1 retinopathy of prematurity: report on fluorescein angiographic findings. Ophthalmology 2014:121:2212-9.

$32 \mathrm{Ng}$ EYJ, Lanigan B, O'Keefe M. Fundus fluorescein angiography in the screening for and management of retinopathy of prematurity. J Pediatr Ophthalmol Strabismus 2006:43:85-90. 